\title{
Identification of the Cartridge Characteristics for HIFU Beauty Care Systems
}

\author{
Jongseok Kim ${ }^{1}$, Hoseung Lee ${ }^{1}$, Jung-Hyeon Jeon ${ }^{1}$ and Chang-Ho Hyun ${ }^{1+}$
}

\begin{abstract}
This paper presents the identification of the cartridge characteristics for HIFU beauty care systems. The stability of the cartridge should be guaranteed because it directly contacts with skin when HIFU beauty care systems are operated. If the stability of the cartridge is not guaranteed, it maybe occur serious unexpected accident. Therefore, there must be a systematic analysis of the cartridge characteristics for HIFU beauty care systems. In order to analyze the stability, its model should be obtained first. Hence, the identification of the cartridge characteristics is studied and its electrical equivalent circuit is proposed. In order to check its validity, some simulation is performed.
\end{abstract}

Keywords - Identification, HIFU, stability, model.

\section{INTRODUCTION}

The interest in maintaining anti-aging appearance has been getting bigger and bigger as the average span of human's life rises. In order to keep the appearance, functional cosmetics for anti-aging have been widely developed and plastic surgery has been prevalent. However, people have been eager for new ways to main the appearance because they want to get quicker results and safer operation [1-3]. Especially, a high intensity focused ultrasound (HIFU) system is very popular since it is a new type of non-surgical, non-invasive procedure.

HIFU is a therapy that destroys targeted tissue with rapid heat elevation [4]. HIFU concentrates high frequency ultrasound waves (similar to a magnifying glass) into an area the size of a grain of rice. At that location, or focal point, the temperature rapidly rises to almost 90 degrees Celsius (195 degrees Fahrenheit). Tissue at the focal point, including cancerous tissue, is destroyed. HIFU uses non-ionizing energy, so the procedure can be repeated, if necessary. HIFU is being studied around the world to treat a wide variety of cancers and soft tissue diseases [5-7].

In the field of beauty care and ageing, HIFU equipment applies high-intensity focused ultrasound energy to locally heat for a lifting result on the skin and uses HIFU for the face's own natural healing process to lift, tone, and tighten loose skin on the brow, neck, under the chin, and even upper chest area.

However, without enough study of the risks that HIFU transducer can occur such as exceeding the desired acoustic power or poor control of tissue targeting, serious problems will be inevitable. To avoid overheating of the active materials in the HIFU transducer, the pulsed signals have been recommended to drive HIFU transducers; For example, driving HIFU 1 minute, shut down 2 minutes for the transducer

1IRS Lab., Dept. of Electrical Electronic and Control Engineering, Kongju National University, Republic of Korea. to cool down. This is too experimental. In order to get reliable solution, the system level identification of transducer, the identification of the cartridge should be systematically sought to ensure safe use. This allows proposals for precautions to be taken during operation to be made.

This type of approach highlights the challenges that must be faced in the immediate future for the development and safe exploitation of HIFU systems. The necessity for standard definitions of the parameters to be checked or monitored during HIFU treatments is crucial, as is the availability of reliable dedicated measurement devices. Since the cartridge of the hand piece concentrates HIFU energy on the skin directly, its reliability should be guaranteed. However, there has been no research about the reliability or stability analysis of the cartridge characteristic with respect to temperature.

This paper presents the identification of the cartridge characteristics for HIFU beauty care systems. The stability of the cartridge should be guaranteed because it directly contacts with skin when HIFU beauty care systems are operated. If the stability of the cartridge is not guaranteed, it maybe occur serious unexpected accident. Therefore, there must be a systematic analysis of the cartridge characteristics for HIFU beauty care systems. In order to analyze the stability, its model should be obtained first. Hence, the identification of the cartridge characteristics is studied and its electrical equivalent circuit is proposed. In order to check its validity, some simulation is performed.

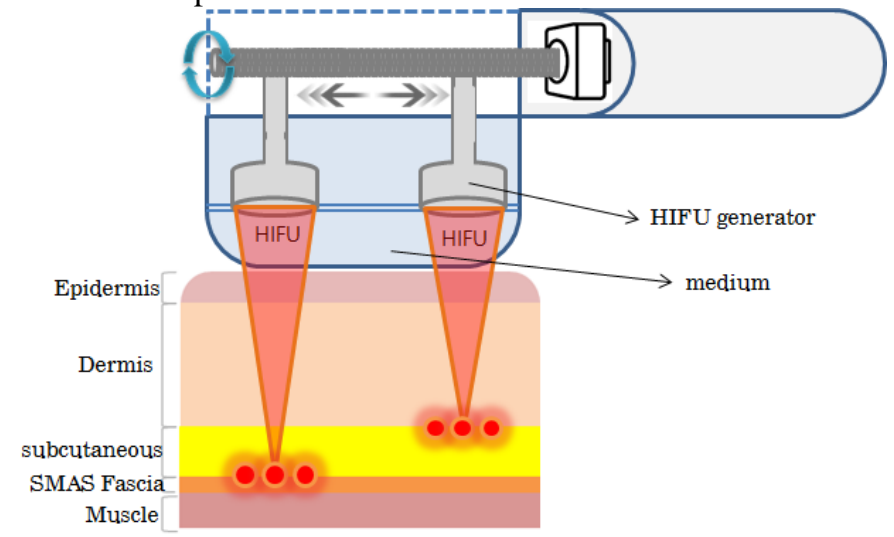

Fig. 1: Structure of the hand piece of HIFU beauty care system

Since the cartridge of the hand piece concentrates HIFU energy on the skin directly, its reliability should be guaranteed. However, there has been no research about the reliability or stability analysis of the cartridge characteristic with respect to temperature. 
This paper presents the identification of the cartridge characteristics for HIFU beauty care systems. The stability of the cartridge should be guaranteed because it directly contacts with skin when HIFU beauty care systems are operated. If the stability of the cartridge is not guaranteed, it maybe occur serious unexpected accident. Therefore, there must be a systematic analysis of the cartridge characteristics for HIFU beauty care systems. In order to analyze the stability, its model should be obtained first. Hence, the identification of the cartridge characteristics is studied and its electrical equivalent circuit is proposed. In order to check its validity, some simulation is performed.

\section{ANALYSIS OF THE CARTRIDGE CHARACTERISTIC}

\section{A. Temperature Analysis of the Medium}

The temperature characteristic of medium in the cartridge, as the ultra-pure water, can be regarded as same as common water. Temperature characteristic of water is nonlinear increase in proportion to the pressure. Fig 2 shows the characteristics of the water. These temperature characteristic in the case of ultra-pure water which is heated by heat generated in the cartridge of the HIFU system also receives the influence of the temperature characteristic.

In order to design a model based controller for the reliability of the cartridge, the temperature characteristic of medium, the ultra-pure water should be modelled. The model can be regarded as the model of the cartridge.

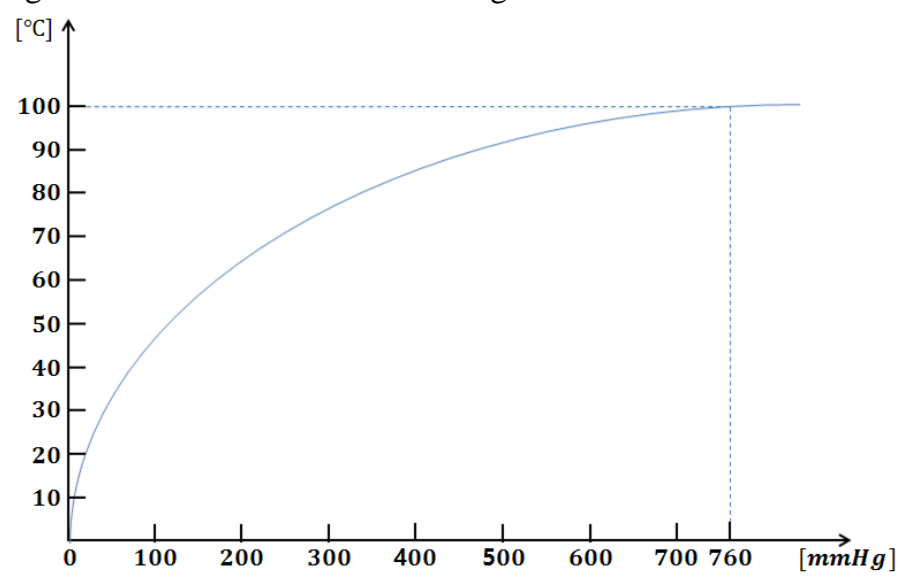

Fig. 2: Characteristic of medium temperature

\section{ELECTRICAL EQUivalent CIRCUIT}

\section{A. Electrical Equivalent Circuit of the Medium Temperature Characteristic}

In order to identify the cartridge, its characteristic shown in fig. 2 will be verified with electrical equivalent model in this section. The electrical model can be constructed by simplifying a serial, R-L circuit as shown in fig.2. R-L-circuit is electrically modelled by Kirchhoff's law as equation (1).

$$
v(t)=\operatorname{Ri}(t)+L \frac{d i(t)}{d t}
$$

Where, $v(t)$ is input voltage, $i(t)$ is current, $R$ is resistance, $L$ is inductance.

From the derivative function (1), the state space model is as follows;

$$
\begin{aligned}
& \dot{x}(t)=-\frac{R}{L} x(t)+\frac{1}{L} u(t) \\
& y(t)=x(t)
\end{aligned}
$$

where, $x(t)=i(t), \quad u(t)=v(t)$.

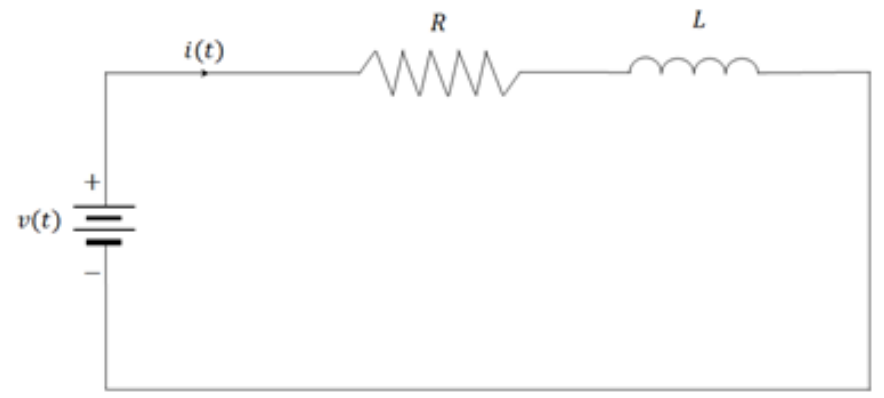

Fig. 3: Electrical model of cartridge.

The input voltage is a sinusoidal function but for the convenience its RMS value are used for simulations. Therefore, the system input can be regarded as DC voltage. DC voltage can be regarded as the step function in Mathematics.

Using Laplace transform, the given equation (1) changes to (3);

$$
V(s)=R I(s)+L s I(s)
$$

From the equation (3), the process to obtain the final value when the input is added to (4)

$$
\frac{1}{s}=R I(s)+\operatorname{Ls} I(s)
$$

Then,

$$
I(s)=\frac{1}{s(R+L s)}
$$

After some calculation (6) can be represented

$$
\begin{aligned}
& I(s)=\frac{1}{R}\left(\frac{1}{S}-\frac{1}{s+R / L}\right) \\
& i(t)=\frac{1}{R}\left(1-e^{-\frac{R}{L} t}\right)
\end{aligned}
$$

\section{Simulation}

In this section, the validity of the proposed model is verified by the comparative analysis with the real characteristic of the cartridge. Fig. 4 shows the temperature change characteristics of the cartridge of the HIFU beauty care system and the instantaneous value of the HIFU beauty care system. As it 
indicates, the response based on the proposed model follows that based on the characteristic of the medium well the error between two responses is not readily recognizable. In fig.5 the error between both of them is presented. There exists the maximum error $0.06^{\circ} \mathrm{C}$ between the response based on the proposed model and that based on the characteristic of the medium. It is a very fine error that. Therefore electrical model of the cartridge presented in the body. In addition, the validity of the proposed model for the possibility as an equivalent model of the temperature characteristics of the medium was verified.

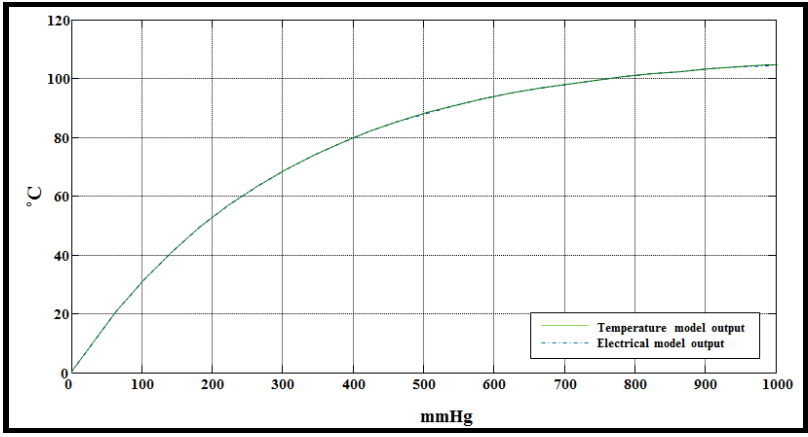

Fig. 4: The comparative simulation result the proposed model with the characteristic of the cartridge

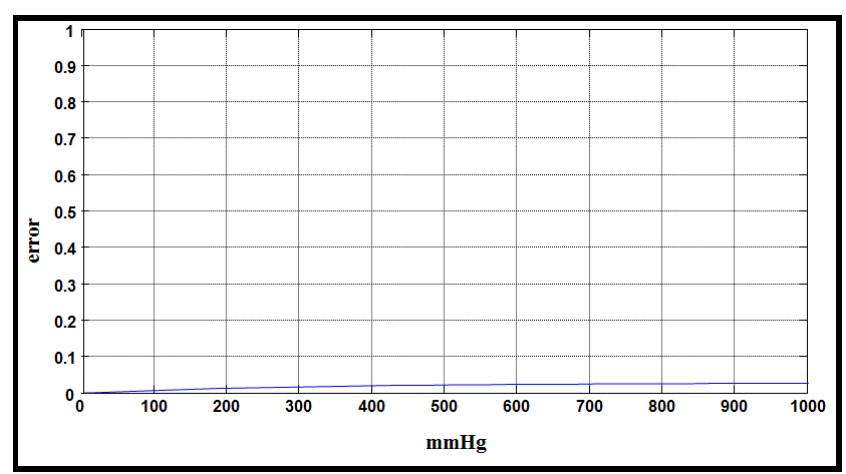

Fig. 5: The temperature error between the proposed model and the characteristic of the cartridge

\section{CONCLUSION}

In this paper, the identification of the cartridge characteristics for HIFU beauty care systems was proposed. In order to derive the model of the HIFU cartridge, the temperature characteristic of the medium was analysed first. Then its electrical equivalent circuit was designed. From the circuit, its model was readily able to be obtained. Some simulation result showed the validity of the proposed model. This model is very useful not only to analyze the stability of the cartridge but also to design a controller to maintain its reliability to use it as a skin care system or an obesity care system. For the future work, the design of controller to control the temperature of the cartridge will be researched since it is very important to keep the temperature steady for the durability of not only the cartridge but also user's safety.

\section{ACKNOWLEDGMENT}

This work (Grants No. C0300644) was supported by Business for Cooperative R\&D between Industry, Academy, and Research Institute funded Korea Small and Medium Business Administration in 2015.

\section{REFERENCES}

[1] S. H. Kim, "High-intensity focused ultrasound return (HIFU) assessment guidelines," The Food and Drug Safety Evaluation, pp.1-12, Oct 2009.

[2] Y. H. Oh, "Trends and entry scheme of beautiful skin equipment market," Kotra, pp. 2-16, Aug 2012.

[3] C. Y. Kim, "Anti-Aging- II -Important my skin, increases management recipient," Market Issue, pp. 1-7, Mar 2015.

[4] W. J. Yu, S. C. Noh, J. H. Park, and H. H. Chio, "A study of heating simulation by HIFU(High Intensity Focused Ultrasound)," The Institute of Electronics Engineers of Korea, pp. 1200-1201, July 2009.

[5] Dhirai Arora, Mikhail Skliar, and Robert b. Roemer, "Model predictive control of ultrasound hyperthermia treatment of cancer," Proceedings of the American Control Conference, pp. 2897-2902, May 2002.

[6] J. A. Jensen, "A new calculation precedure for spatial impulse responses in ultrasound," In press, pp. 3266-3274, Oct 1999.

[7] J. A. Jensen, D. Gandhi, and W. D. O'Brien. "Ultrasound field in and attenuating medium. In Proc. IEEE Ultrason. Symp., pages 943-946, 1993.

http://dx.doi.org/10.1109/ULTSYM.1993.339652 